\title{
History-Sensitive Accumulation Rules for Life-Time Prediction under Variable Creep Loading
}

\author{
S.E. Mikhailov*, I.V. Namestnikova \\ Brunel University West London, UK
}

\begin{abstract}
A general form of temporal strength conditions under variable creep loading is employed to formulate several new phenomenological accumulation rules based on the constant-loading durability diagram. Unlike the well-known Robinson rule of linear accumulation of partial life-times, the new rules allow to describe the life-time sensibility to the load sequence, observed in experiments. Comparison of one of the new rules with experimental data shows that it fits the data much more accurately than the Robinson rule.
\end{abstract}

Keywords: Temporal strength, Life time, Accumulation rules, Creep, Load sequence, History dependence

\section{Introduction}

Let $\boldsymbol{\sigma}=\sigma_{i j}$ be the stress tensor and let us consider a time-dependent loading processes $\boldsymbol{\sigma}(\tau)$ starting at time $\tau=0$ (i.e., $\boldsymbol{\sigma}(\tau)=0$ at $\tau<0$ ) and then generally varying in time at $\tau \geq 0$. Many materials at elevated temperatures and some materials at room temperature exhibit dependence of their strength not only on the level of applied loading at a particular instant but also on the duration of load application, and generally on the history of loading.

Let $t^{* 0}(\boldsymbol{\sigma})$ be a material durability (life-time) under a constant stress state $\boldsymbol{\sigma}=$ const, at $\tau \geq 0$. For uniaxial constant loading the dependence of the durability on applied load $t^{* 0}(\sigma)$, or vice-versa, temporal strength on the load duration $\sigma^{* 0}(t)$ is usually drown as the durability curve (or durability diagram). Some schematized examples of the durability diagrams are shown in Figs. 1-3. Such diagrams are obtained experimentally for many materials and allow easily to predict the material life time under the constant (at $\tau \geq 0$ ) applied load. Evidently, the durability diagrams can also be obtained for multiaxial constat stresses $\boldsymbol{\sigma}$.

However, if the applied stress is not a constant at $\tau \geq 0$ but a general function of time, i.e., process $\boldsymbol{\sigma}(\tau)$, then one has to have a model to predict the life-time under variable lading from the available durability diagram obtained under constant loads. One of the most popular is the Robinson model (rule) of linear accumulation of partial life-times, (Robinson, 1938, 1952) (see also e.g. Rabotnov, 1969; Penny and

${ }^{*}$ Corresponding author, E-mail: sergey.mikhailov@brunel.ac.uk,

Dept. of Mathematical Sciences, John Crank Building, Brunel University West London, Uxbridge, UB8 3PH, UK, Tel: +44 (0)1895 267361, Fax: +44 (0)1895 269732 
Marriott, 1971), which allows to calculate the life-time $t^{*}=t^{*}(\boldsymbol{\sigma})$ under a variable process $\boldsymbol{\sigma}(\tau)$ from the durability diagram under constant loads, $t^{* 0}(\boldsymbol{\sigma})$, by equation

$$
\int_{0}^{t^{*}} \frac{d \tau}{t^{* 0}(\boldsymbol{\sigma}(\tau))}=1 .
$$

The well-known non-sensitivity of the Robinson model to the sequence of load application can be readily observed from (1): applying first higher and then lower load or vice-versa lead to the same life-time. However many experiments on variable loading show that this is generally not the case. Although some modifications of the Robinson model to address this issue have been reported in the literature, see e.g. (Bui-Quoc 1972, Lokoshchenko and Namestnikova 1983, Gomuc and Bui-Quoc 1986) and references therein, no one seems to be widely accepted.

In this paper we propose new linear and non-linear accumulation rules (NES accumulation rules), based on the concept of Normalised Equivalent Stress, for processes under constant temperature. Similar to the Robinson rule, the NES rules use the durability diagram under constant load as entry data, but unlike the Robinson rule, they are sensitive to the load history. It is demonstrated that the linear NES rule much better fits some experimental results than the Robinson rule. Moreover, it is shown that the linear NES rule is able to recover several known accumulation rules from the durability diagram under constant load obtained from them. This develops some preliminary results presented in (Mikhailov and Namestnikova, 2009).

\section{Normalised equivalent stress functional}

To propose a new accumulation rule sensible to the load sequence, we first remark that any model for time-dependent strength and life-time analysis under constant temperature leads to the temporal strength condition in the form

$$
\underline{\Lambda}^{T}(\boldsymbol{\sigma} ; t)<1
$$

with the corresponding temporal rupture criterion $\underline{\Lambda}^{T}\left(\boldsymbol{\sigma} ; t^{*}\right)=1$. Here $\underline{\Lambda}^{T}(\boldsymbol{\sigma} ; t)$ is value of the temporal normalised equivalent stress functional, NESF. Its rigorous definition and properties are given in (Mikhailov, 2003), see also Appendix. For many practical situations $\underline{\Lambda}^{T}(\boldsymbol{\sigma} ; t)$ can be understood as the number $\Lambda$ such that rupture under the scaled process $\sigma_{i j}(\tau) / \Lambda$ happens just at time $t$. The NESF $\underline{\Lambda}^{T}(\boldsymbol{\sigma} ; t)$ can be also perceived as an experimentally measurable damage parameter, unlike many other damage measures that are non-measurable internal variables.

Let us consider some examples of normalised equivalent stresses, which (except the third one) were analysed in (Mikhailov, 2003), for some well known temporal strength conditions under a non-negative uniaxial stress process $\sigma(\tau)$ starting at $\tau=0$. Although the first two of the four example NESFs are used mostly in dynamic (i.e. short-time) strength, their qualitative features are similar to the NESFs in creep (i.e. long-time) strength. Moreover, all the analytical reasoning presented in the paper in terms of the creep strength can be equally applied to the dynamic strength analysis. 
The Nikiforovski-Shemyakin dynamic strength condition (Nikiforovsky, 1976; Nikiforovsky and Shemyakin, 1979).

$$
\int_{0}^{t} \sigma(\tau) d \tau<I_{c}
$$

where $I_{c}>0$ is a material parameter. Then in terms of NESF it can be rewritten as

$$
\underline{\Lambda}^{N S}(\sigma ; t)=\frac{1}{I_{c}} \int_{0}^{t} \sigma(\tau) d \tau<1
$$

The incubation time strength condition, see (Morozov and Petrov, 2000; Kashtanov and Petrov, 2007; Kashtanov, Petrov, Pugno and Carpinteri 2008), modified as in (Mikhailov 2003, Section 6.3), has the following form

$$
\frac{1}{t_{c}} \int_{t^{\prime}-t_{c}}^{t^{\prime}} \sigma(\tau) d \tau<\sigma_{c} \text { for all } t^{\prime} \leq t
$$

where $t_{c}>0$ and $\sigma_{c}>0$ are material parameters. In terms of NESF it can be rewritten as

$$
\underline{\Lambda}^{I T}(\sigma ; t)=\frac{1}{\sigma_{c} t_{c}} \max _{t^{\prime} \leq t^{\prime}} \int_{t^{\prime}-t_{c}}^{t} \sigma(\tau) d \tau<1 .
$$

The Abel type strength condition, see Suvorova (1979), Suvorova, Viktorova, Mashinskaya (1980), has the following form, involving the Abel type integral operator,

$$
\sigma\left(t^{\prime}\right)+(1-\alpha) \kappa \int_{0}^{t^{\prime}} \frac{\sigma(\tau) d \tau}{\left(t^{\prime}-\tau\right)^{\alpha}}<\sigma_{c} \quad \text { for all } t^{\prime} \leq t
$$

where $\kappa>0, \alpha<1$ and $\sigma_{c}>0$ are material parameters. In terms of NESF it can be rewritten as

$$
\underline{\Lambda}^{A}(\sigma ; t)=\frac{1}{\sigma_{c}} \max _{t^{\prime} \leq t}\left\{\sigma\left(t^{\prime}\right)+(1-\alpha) \kappa \int_{0}^{t^{\prime}} \frac{\sigma(\tau)}{\left(t^{\prime}-\tau\right)^{\alpha}} d \tau\right\}<1 .
$$

The temporal strength condition associated with the Robinson rule can also be re-written in the form

$$
\underline{\Lambda}^{T R}(\sigma ; t)<1
$$

where $\underline{\Lambda}^{T R}(\sigma ; t)$ is the solution (the minimal one if there are several solutions) $\Lambda$ of the equation

$$
\int_{0}^{t^{*}} \frac{d \tau}{t^{* 0}(\sigma(\tau) / \Lambda)}=1
$$

In Section 5 we give an explicit solution of this equation leading to an explicit expression for $\underline{\Lambda}^{T R}$ obtained in (Mikhailov, 2003) for the power-type durability diagram. 


\section{Some NES accumulation rules}

\subsection{Motivation}

The temporal strength condition under constant uniaxial loading $\sigma=$ const starting at $t=0$ can be written as $|\sigma|<\sigma^{* 0}(t)$, where the function $\sigma^{* 0}(t)$ gives another form of the durability diagram and is inverse to the function $t^{* 0}(\sigma)$, i.e. the equality $|\sigma|=\sigma^{* 0}\left(t^{* 0}(\sigma)\right)$ is identically satisfied for any $\sigma$. Similarly, the temporal strength condition under constant multiaxial loading $\boldsymbol{\sigma}=$ const started at $t=0$, can be written as $|\boldsymbol{\sigma}|<\sigma^{* 0}(\tilde{\boldsymbol{\sigma}} ; t)$, which can be reformulated in terms of the NESF as $\underline{\Lambda}^{T}(\boldsymbol{\sigma} ; t)<1$, where

$$
\underline{\Lambda}^{T}(\boldsymbol{\sigma} ; t):=\frac{|\boldsymbol{\sigma}|}{\sigma^{* 0}(\tilde{\boldsymbol{\sigma}} ; t)}
$$

Here $|\boldsymbol{\sigma}|$ is a matrix norm of the tensor $\boldsymbol{\sigma}$, e.g., $|\boldsymbol{\sigma}|=\sqrt{\sum_{i, j=1}^{3} \sigma_{i j} \sigma_{i j}}$, and $\tilde{\boldsymbol{\sigma}}:=\boldsymbol{\sigma} /|\boldsymbol{\sigma}|$ is the unit tensor presenting the stress tensor $\boldsymbol{\sigma}$ shape. Making formal manipulations, we have from (10),

$$
\begin{aligned}
\underline{\Lambda}^{T}(\boldsymbol{\sigma} ; t) & =\frac{|\boldsymbol{\sigma}|}{\sigma^{* 0}(\tilde{\boldsymbol{\sigma}} ; 0)}+\int_{0}^{t}|\boldsymbol{\sigma}| \frac{\partial}{\partial \xi}\left[\frac{1}{\sigma^{* 0}(\tilde{\boldsymbol{\sigma}} ; \xi)}\right] d \xi \\
= & \frac{|\boldsymbol{\sigma}|}{\sigma^{* 0}(\tilde{\boldsymbol{\sigma}} ; 0)}+\int_{0}^{t}|\boldsymbol{\sigma}| \frac{\partial}{\partial t}\left[\frac{1}{\sigma^{* 0}(\tilde{\boldsymbol{\sigma}} ; t-\tau)}\right] d \tau=\frac{d}{d t} \int_{0}^{t} \frac{|\boldsymbol{\sigma}|}{\sigma^{* 0}(\tilde{\boldsymbol{\sigma}} ; t-\tau)} d \tau .
\end{aligned}
$$

\subsection{Linear NES accumulation rule}

Hinted by (11), we extend the form for $\underline{\Lambda}^{T}$ also to variable loading $\sigma_{i j}(\tau)$, such that $\sigma_{i j}(\tau)=0$ at $\tau<0$, introducing the following strength condition,

$$
\underline{\Lambda}_{1}^{T S}(\boldsymbol{\sigma} ; t):=\max _{t^{\prime} \leq t} \underline{\hat{\Lambda}}_{1}^{T S}\left(\boldsymbol{\sigma} ; t^{\prime}\right)<1
$$

where the value $\underline{\hat{\Lambda}}_{1}^{T S}\left(\boldsymbol{\sigma} ; t^{\prime}\right)$, which will be further called the normalised equivalent stress, is defined as

$$
\begin{aligned}
\underline{\Lambda}_{1}^{T S}\left(\boldsymbol{\sigma} ; t^{\prime}\right) & :=\frac{d}{d t^{\prime}} \int_{0}^{t^{\prime}} \frac{|\boldsymbol{\sigma}(\tau)|}{\sigma^{* 0}\left(\tilde{\boldsymbol{\sigma}}(\tau) ; t^{\prime}-\tau\right)} d \tau \\
& =\frac{\left|\boldsymbol{\sigma}\left(t^{\prime}\right)\right|}{\sigma^{* 0}\left(\tilde{\boldsymbol{\sigma}}\left(t^{\prime}\right) ; 0\right)}+\int_{0}^{t^{\prime}}|\boldsymbol{\sigma}(\tau)| \frac{\partial}{\partial t^{\prime}}\left[\frac{1}{\sigma^{* 0}\left(\tilde{\boldsymbol{\sigma}}(\tau) ; t^{\prime}-\tau\right)}\right] d \tau \\
& =\int_{-0}^{t^{\prime}}\left\{\frac{\partial}{\partial \tau}\left[\frac{|\boldsymbol{\sigma}(\tau)|}{\sigma^{* 0}(\tilde{\boldsymbol{\sigma}}(\tau) ; \eta)}\right]\right\}_{\eta=t^{\prime}-\tau} d \tau .
\end{aligned}
$$

The last equality in (13) is obtained by integration by parts and the derivative there should be understood in the generalised sense, i.e., expressed in terms of the Dirac delta-function at the finite jumps of the processes $\boldsymbol{\sigma}(t)$, and the integral then is understood also in the generalised sense. It is assumed that $\boldsymbol{\sigma}(-0)$ is zero at the 
lover integration limit $\tau=-0$, to handle the step-like loading $\boldsymbol{\sigma}(\tau)$ jumping from 0 to a finite value at $\tau=0$.

Not that $\underline{\Lambda}_{1}^{T S}\left(\boldsymbol{\sigma} ; t^{\prime}\right)$ may be non-monotonous in $t^{\prime}$ while $\underline{\Lambda}_{1}^{T S}(\boldsymbol{\sigma} ; t)$ is non-decreasing in $t$. The maximum in (12) and in similar formulas further on should be replaced with supremum if $\underline{\Lambda}_{1}^{T S}\left(\boldsymbol{\sigma} ; t^{\prime}\right)$ is discontinuous in $t^{\prime}$. We will show in the following that relations (12)-(13) can be interpreted as a linear accumulation rule for partial NES, i.e., an alternative counterpart of the Robinson rule of linear accumulation of partial life-times.

For constant loadings started at $t=0$, the NES accumulation rule (12)-(13), by its deduction above, coincides exactly with predictions of the durability diagram employed in it, as well as the Robinson rule does. However the memory-type dependence on the difference $t^{\prime}-\tau$ in (13) produces a sensitivity to load history, which is absent in the Robinson linear accumulation rule. Note that some other temporal strength conditions involving the memory-type dependence on $t^{\prime}-\tau$ were suggested by Il'ushin (1967), see also (Mikhailov, 2003, Section 6.6).

If the durability diagram can be presented as a product,

$$
\sigma^{* 0}(\tilde{\boldsymbol{\sigma}} ; t)=\sigma^{0}(\tilde{\boldsymbol{\sigma}}) \sigma^{* *}(t),
$$

where the scalar functions $\sigma^{0}$ and $\sigma^{* *}$ are material characteristics, then (13) simplifies to

$$
\begin{aligned}
\underline{\Lambda}_{1}^{T S}\left(\boldsymbol{\sigma} ; t^{\prime}\right) & =\frac{\left|\boldsymbol{\sigma}\left(t^{\prime}\right)\right|}{\sigma^{0}\left(\tilde{\boldsymbol{\sigma}}\left(t^{\prime}\right)\right) \sigma^{* *}(0)}-\int_{0}^{t^{\prime}} \frac{|\boldsymbol{\sigma}(\tau)|}{\sigma^{0}(\tilde{\boldsymbol{\sigma}}(\tau))} \frac{\partial}{\partial \tau}\left[\frac{1}{\sigma^{* *}\left(t^{\prime}-\tau\right)}\right] d \tau \\
& =\int_{-0}^{t^{\prime}} \frac{1}{\sigma^{* *}\left(t^{\prime}-\tau\right)} d\left[\frac{|\boldsymbol{\sigma}(\tau)|}{\sigma^{0}(\tilde{\boldsymbol{\sigma}}(\tau))}\right] .
\end{aligned}
$$

Here the last integral should be understood in the Stiltjes sense, i.e., the differential should be understood as the corresponding jump at the points of discontinuity of the function $\boldsymbol{\sigma}(t)$ (if there are any). For example, if $\boldsymbol{\sigma}(t)=0$ for $t<0$ and $\boldsymbol{\sigma}(t)=\boldsymbol{\sigma}_{1}=$ const for $t \geq 0$, then (15) gives

$$
\hat{\Lambda}_{1}^{T S}\left(\boldsymbol{\sigma} ; t^{\prime}\right)=\frac{1}{\sigma^{* *}\left(t^{\prime}\right)}\left[\frac{|\boldsymbol{\sigma}(+0)|}{\sigma^{0}(\tilde{\boldsymbol{\sigma}}(+0))}-\frac{|\boldsymbol{\sigma}(-0)|}{\sigma^{0}(\tilde{\boldsymbol{\sigma}}(-0))}\right]=\frac{1}{\sigma^{* *}\left(t^{\prime}\right)} \frac{\left|\boldsymbol{\sigma}_{1}\right|}{\sigma^{0}\left(\tilde{\boldsymbol{\sigma}}_{1}\right)}=\frac{\left|\boldsymbol{\sigma}_{1}\right|}{\sigma^{* 0}\left(\tilde{\boldsymbol{\sigma}}_{1} ; t^{\prime}\right)},
$$

which coincides with (10), as expected.

Relation (15) simplifies further if we apply it to an in-phase (coaxial) loading, with the components of the tensor $\boldsymbol{\sigma}(\tau)$ not changing sign (e.g. a uniaxial loading $\sigma(\tau)$ without change of sign). Then $\tilde{\boldsymbol{\sigma}}=$ const and one can take $\sigma^{0}(\tilde{\boldsymbol{\sigma}})=1$ in (14) and (15), i.e., take $\sigma^{* 0}(\tilde{\boldsymbol{\sigma}} ; t)=\sigma^{* *}(t)$. This reduces (13) to the following simplified form of the linear accumulation rule for the notional NES,

$$
\underline{\Lambda}_{1}^{T S}\left(\boldsymbol{\sigma} ; t^{\prime}\right)=\frac{d}{d t^{\prime}} \int_{0}^{t^{\prime}} \frac{|\boldsymbol{\sigma}(\tau)|}{\sigma^{* *}\left(t^{\prime}-\tau\right)} d \tau=\int_{-0}^{t^{\prime}} \frac{d|\boldsymbol{\sigma}(\tau)|}{\sigma^{* *}\left(t^{\prime}-\tau\right)} .
$$

Let us consider a step-wise loading

$$
\boldsymbol{\sigma}(\tau)= \begin{cases}0, & \tau<t_{0}=0 \\ \boldsymbol{\sigma}_{k}, & t_{k-1} \leq \tau<t_{k}, \quad 0<k\end{cases}
$$


If $\tilde{\boldsymbol{\sigma}}=$ const., i.e. the shape tensor is the same on all steps, then (16) becomes

$$
\underline{\hat{\Lambda}}_{1}^{T S}\left(\boldsymbol{\sigma} ; t^{\prime}\right)=\sum_{0 \leq t_{k}<t^{\prime}} \frac{\left|\boldsymbol{\sigma}\left(t_{k}+0\right)\right|-\left|\boldsymbol{\sigma}\left(t_{k}-0\right)\right|}{\sigma^{* *}\left(t^{\prime}-t_{k}\right)} .
$$

This means that the total NES accumulates the partial NESes produced by the changes of the stress tensor and associated with the durability diagram started at the change instant.

In the general case of step-wise loading, i.e., when $\tilde{\boldsymbol{\sigma}} \neq$ const. in (17), expression (13) gives the following counterpart of (18),

$$
\underline{\Lambda}_{1}^{T S}\left(\boldsymbol{\sigma} ; t^{\prime}\right)=\sum_{0 \leq t_{k}<t^{\prime}}\left[\frac{\left|\boldsymbol{\sigma}\left(t_{k}+0\right)\right|}{\sigma^{* 0}\left(\tilde{\boldsymbol{\sigma}}\left(t_{k}+0\right), t^{\prime}-t_{k}\right)}-\frac{\left|\boldsymbol{\sigma}\left(t_{k}-0\right)\right|}{\sigma^{* 0}\left(\tilde{\boldsymbol{\sigma}}\left(t_{k}-0\right), t^{\prime}-t_{k}\right)}\right]
$$

that can be still interpreted as the linear accumulation rule for partial NESes.

\subsection{Non-linear NES accumulation rules}

By the argument similar to the derivation of the linear NES rule, one can also arrive at a more general non-linear (power-type) rule of NES accumulation, leading to the following strength condition,

$$
\underline{\Lambda}_{\beta}^{T S}(\boldsymbol{\sigma} ; t):=\max _{t^{\prime} \leq t} \underline{\Lambda}_{\beta}^{T S}\left(\boldsymbol{\sigma} ; t^{\prime}\right)<1
$$

with the nonlinear NES

$$
\begin{aligned}
\underline{\Lambda}_{\beta}^{T S}\left(\boldsymbol{\sigma} ; t^{\prime}\right) & :=\left[\frac{d}{d t^{\prime}} \int_{0}^{t^{\prime}}\left|\frac{\boldsymbol{\sigma}(\tau)}{\sigma^{* 0}\left(\tilde{\boldsymbol{\sigma}}(\tau) ; t^{\prime}-\tau\right)}\right|^{\beta} d \tau\right]^{1 / \beta} \\
& =\left[\int_{-0}^{t^{\prime}}\left\{\frac{\partial}{\partial \tau}\left(\left|\frac{\boldsymbol{\sigma}(\tau)}{\sigma^{* 0}(\tilde{\boldsymbol{\sigma}}(\tau) ; \eta)}\right|^{\beta}\right)\right\}_{\eta=t^{\prime}-\tau} d \tau\right]^{1 / \beta},
\end{aligned}
$$

where $\beta>0$ is a material parameter. Under condition (14), expression (21) reduces to

$$
\underline{\hat{\Lambda}}_{\beta}^{T S}\left(\boldsymbol{\sigma} ; t^{\prime}\right)=\left[\int_{-0}^{t^{\prime}} \frac{1}{\left[\sigma^{* *}\left(t^{\prime}-\tau\right)\right]^{\beta}} d\left(\left|\frac{\boldsymbol{\sigma}(\tau)}{\sigma^{0}(\tilde{\boldsymbol{\sigma}}(\tau))}\right|^{\beta}\right)\right]^{1 / \beta} .
$$

For a constant multiaxial loading $\sigma_{i j}=$ const started at $t=0$, expression (21) reduces to $(10)$ for any $\beta$. In the general case of step-wise multiaxial loading, expression (21) gives,

$$
\underline{\Lambda}_{1}^{T S}\left(\boldsymbol{\sigma} ; t^{\prime}\right)=\left[\sum_{0 \leq t_{k}<t^{\prime}}\left(\left|\frac{\boldsymbol{\sigma}\left(t_{k}+0\right)}{\sigma^{* 0}\left(\tilde{\boldsymbol{\sigma}}\left(t_{k}+0\right), t^{\prime}-t_{k}\right)}\right|^{\beta}-\left|\frac{\boldsymbol{\sigma}\left(t_{k}-0\right)}{\sigma^{* 0}\left(\tilde{\boldsymbol{\sigma}}\left(t_{k}-0\right), t^{\prime}-t_{k}\right)}\right|^{\beta}\right)\right]^{1 / \beta}
$$


that can be interpreted as a nonlinear accumulation rule for partial NES. The parameter $\beta$ influences the sign and the degree of the deviation from the Robinson accumulation rule, cf. Sections 5,6 .

One can see that a linear combination of the nonlinear terms (21) leads to a strength condition based on even more general non-linear rule of NES accumulation,

$$
\begin{gathered}
\underline{\Lambda}_{m u l t}^{T S}(\boldsymbol{\sigma} ; t):=\max _{t^{\prime} \leq t} \underline{\hat{\Lambda}}_{m u l t}^{T S}\left(\boldsymbol{\sigma} ; t^{\prime}\right)<1, \\
\underline{\hat{\Lambda}}_{m u l t}^{T S}\left(\boldsymbol{\sigma} ; t^{\prime}\right):=\sum_{n=1}^{N} \alpha_{n} \underline{\hat{\Lambda}}_{\beta_{n}}^{T S}\left(\boldsymbol{\sigma} ; t^{\prime}\right),
\end{gathered}
$$

where the numbers $\beta_{n}>0$ and $\alpha_{n}$ are material parameters with $\sum_{n}^{N} \alpha_{n}=1$, to ensure that $\underline{\Lambda}_{\text {mult }}^{\text {TS }}(\boldsymbol{\sigma} ; t)$ reduces to $(10)$ for $\boldsymbol{\sigma}=$ const started at $t=0$. Such multi-parameter accumulation rule may be useful for the materials for which one parameter $\beta$ does not suffice to fit the experimental data, or when contribution of several durability mechanisms are to be taken into account. However we will not use the multi-parameter rule further in this paper.

Note that for fatigue some counterparts of the accumulation rules presented in this section were given in (Mikhailov and Namestnikova, 2003, 2004).

\section{Recovery of accumulation rules from correspond- ing durability diagrams}

Let us validate the Robinson and NES accumulation rules against their ability to recover the known temporal strength conditions mentioned in Section 2 from their corresponding durability diagrams.

\subsection{Recovery of the Nikiforovski-Shemyakin temporal strength condition}

Substituting the stress, which is constant at $\tau \geq 0$ in the Nikiforovski-Shemyakin strength condition (3), we obtain the following two forms of the associated durability diagram illustrated by Fig. 1,

$$
\sigma^{* 0}(t)=\frac{I_{c}}{t}, \quad t^{* 0}(\sigma)=\frac{I_{c}}{\sigma} .
$$

Let now $\sigma(\tau) \geq 0$ be an arbitrary function. Then strength condition corresponding to the Robinson rule (1) and the second relation in (26) give,

$$
\int_{0}^{t^{*}} \frac{d \tau}{t^{* 0}(\sigma(\tau))}=\frac{1}{I_{c}} \int_{0}^{t^{*}} \sigma(\tau) d \tau<1,
$$

which coincides with (3). 


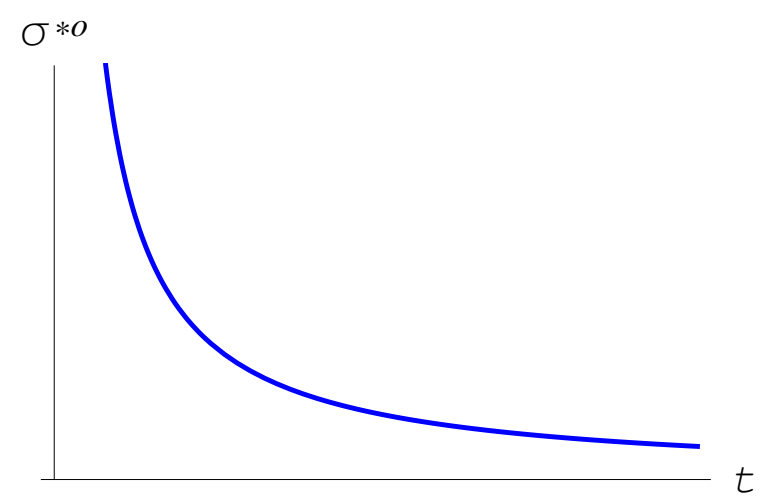

Figure 1: Durability diagram for the Nikiforovski-Shemyakin strength condition.

On the other hand, the strength condition (2), the linear NES accumulation rule (13) and the first relation in (26) give,

$$
\begin{aligned}
\underline{\Lambda}_{1}^{T S}(\sigma ; t)=\max _{t^{\prime} \leq t} \underline{\Lambda}_{1}^{T S}\left(\sigma ; t^{\prime}\right)= & \max _{t^{\prime} \leq t} \frac{d}{d t^{\prime}} \int_{0}^{t^{\prime}} \frac{|\sigma(\tau)|}{\sigma^{* 0}\left(t^{\prime}-\tau\right)} d \tau \\
& =\max _{t^{\prime} \leq t} \frac{d}{d t^{\prime}} \int_{0}^{t^{\prime}} \frac{\left(t^{\prime}-\tau\right) \sigma(\tau)}{I_{c}} d \tau=\frac{1}{I_{c}} \int_{0}^{t} \sigma(\tau) d \tau<1,
\end{aligned}
$$

which also coincides with (3).

Thus both, the Robinson accumulation rule and the NES linear accumulation rule, successfully recover the Nikiforovski-Shemyakin strength condition (3) from its durability diagram (26).

\subsection{Recovery of the incubation time strength condition}

Substituting the stress, which is constant at $\tau \geq 0$ in the incubation time strength condition (5), we obtain the following two forms of the associated durability diagram with the threshold $\sigma_{c}$ (see Fig. 2),

$$
\sigma^{* 0}(t)=\left\{\begin{array}{ll}
\frac{t_{c} \sigma_{c}}{t}, & 0 \leq t \leq t_{c} \\
\sigma_{c}, & 0 \leq t_{c}<t
\end{array} \quad, \quad t^{* 0}(\sigma)= \begin{cases}\infty, & 0 \leq|\sigma| \leq \sigma_{c} \\
\frac{t_{c} \sigma_{c}}{\sigma}, & 0 \leq \sigma_{c}<|\sigma|\end{cases}\right.
$$

Let now $\sigma(\tau) \geq 0$ be an arbitrary function. Then strength condition corresponding to the Robinson rule (1) with the second relation in (27) gives,

$$
\int_{0}^{t^{*}} \frac{d \tau}{t^{* 0}(\sigma(\tau))}=\int_{0}^{t^{*}} \frac{\sigma(\tau) H\left[\sigma(\tau)-\sigma_{c}\right] d \tau}{t_{c} \sigma_{c}}<1
$$

where $H[x]=\left\{\begin{array}{ll}x, & \text { for } x>0 \\ 0, & \text { for } x \leq 0\end{array}\right.$ is the Heaviside function. This condition does not coincide with (5). E.g., for the loading process linear in time, $\sigma(\tau)=\sigma_{c} \tau / t_{c}$ the Robinson rule (28) gives $t<\sqrt{3} t_{c}$, while (5) gives $t<3 t_{c} / 2$. 


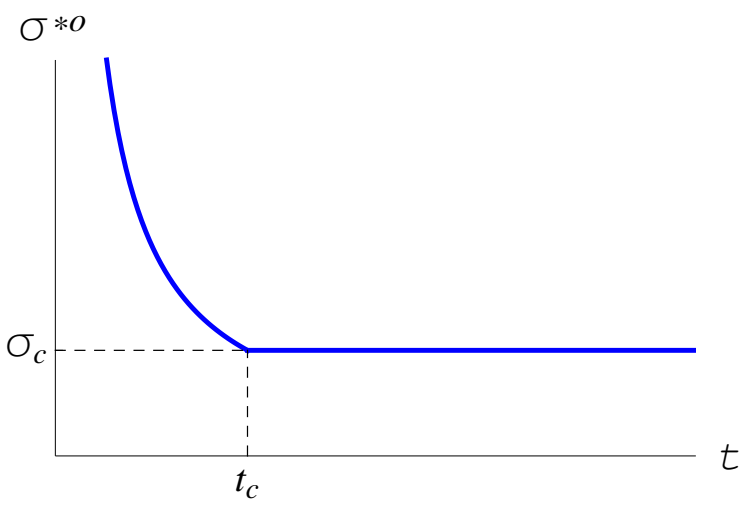

Figure 2: Durability diagram for the incubation time strength condition

On the other hand, the strength condition (2), the linear NES accumulation rule (13) and the first relation in (27) give, if we take into account that $\sigma(\tau)=0$ for $\tau<0$,

$$
\begin{aligned}
\underline{\Lambda}_{1}^{T S}(\sigma ; t)= & \max _{t^{\prime} \leq t} \hat{\Lambda}_{1}^{T S}\left(\sigma ; t^{\prime}\right)=\max _{t^{\prime} \leq t} \frac{d}{d t^{\prime}} \int_{0}^{t^{\prime}} \frac{|\sigma(\tau)|}{\sigma^{* 0}\left(t^{\prime}-\tau\right)} d \tau \\
& =\max _{t^{\prime} \leq t} \frac{d}{d t^{\prime}}\left\{\frac{1}{\sigma_{c}} \int_{-\infty}^{t^{\prime}-t_{c}} \sigma(\tau) d \tau+\frac{1}{t_{c} \sigma_{c}} \int_{t^{\prime}-t_{c}}^{t^{\prime}}\left(t^{\prime}-\tau\right) \sigma(\tau) d \tau\right\} \\
& =\max _{t^{\prime} \leq t}\left\{\frac{\sigma\left(t^{\prime}-t_{c}\right)}{\sigma_{c}}-\frac{t_{c} \sigma\left(t^{\prime}-t_{c}\right)}{t_{c} \sigma_{c}}+\frac{1}{t_{c} \sigma_{c}} \int_{t^{\prime}-t_{c}}^{t^{\prime}} \sigma(\tau) d \tau\right\} \\
& =\frac{1}{t_{c} \sigma_{c}} \max _{t^{\prime} \leq t} \int_{t^{\prime}-t_{c}}^{t^{\prime}} \sigma(\tau) d \tau<1,
\end{aligned}
$$

which coincides with (6).

\subsection{Recovery of the Abel type strength condition}

Substituting the stress, which is constant at $\tau \geq 0$ in the Abel type strength condition (7), we obtain the following two forms of the associated durability diagram possessing a finite instant strength $\sigma_{c}$, illustrated by Fig. 3,

$$
\sigma^{* 0}(t)=\frac{\sigma_{c}}{1+\kappa t^{1-\alpha}}, \quad t^{* 0}(\sigma)=\left[\left(\frac{\sigma_{c}}{\sigma}-1\right) \frac{1}{\kappa}\right]^{\frac{1}{1-\alpha}}
$$

Let now $\sigma(\tau) \geq 0$ be an arbitrary function. Then strength condition corresponding to the Robinson rule (1) with the second relation in (29) gives,

$$
\int_{0}^{t^{*}} \frac{d \tau}{t^{* 0}(\sigma(\tau))}=\int_{0}^{t^{*}}\left[\left(\frac{\sigma_{c}}{\sigma(\tau)}-1\right) \frac{1}{\kappa}\right]^{-\frac{1}{1-\alpha}} d \tau<1
$$




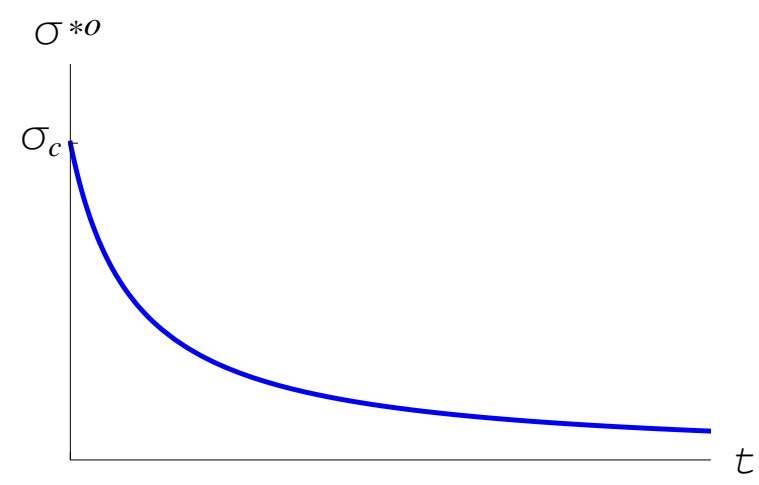

Figure 3: Durability diagram for the Abel type strength condition

which does not coincide with (7). E.g., for the loading process linear in time, $\sigma(\tau)=$ $\sigma_{c} \kappa^{\frac{1}{1-\alpha}} \tau$, and $\alpha=1 / 2$, the numerical calculations show that the Robinson rule (30) gives $t<0.752 \kappa^{-\frac{1}{1-\alpha}}$, while $(7)$ gives $t<0.650 \kappa^{-\frac{1}{1-\alpha}}$.

On the other hand, the NES strength condition (2), the linear NES accumulation rule (13) and the first relation in (29) give, if we take into account that $\sigma(\tau)=0$ for $\tau<0$

$$
\begin{array}{r}
\underline{\Lambda}_{1}^{T S}(\sigma ; t)=\max _{t^{\prime} \leq t} \underline{\hat{\Lambda}}_{1}^{T S}\left(\sigma ; t^{\prime}\right)=\max _{t^{\prime} \leq t}\left\{\frac{\left|\sigma\left(t^{\prime}\right)\right|}{\sigma^{* 0}(0)}+\int_{-0}^{t^{\prime}}|\sigma(\tau)| \frac{\partial}{\partial t^{\prime}}\left[\frac{1}{\sigma^{* 0}\left(t^{\prime}-\tau\right)}\right] d \tau\right\}= \\
\max _{t^{\prime} \leq t}\left\{\frac{\sigma\left(t^{\prime}\right)}{\sigma_{c}}+\int_{-0}^{t^{\prime}} \sigma(\tau) \frac{\partial}{\partial t^{\prime}}\left[\frac{1+\kappa\left(t^{\prime}-\tau\right)^{1-\alpha}}{\sigma_{c}}\right] d \tau\right\}= \\
\frac{1}{\sigma_{c}} \max _{t^{\prime} \leq t}\left\{\sigma\left(t^{\prime}\right)+(1-\alpha) \kappa \int_{0}^{t^{\prime}} \frac{\sigma(\tau)}{\left(t^{\prime}-\tau\right)^{\alpha}} d \tau\right\}<1,
\end{array}
$$

which coincides with (8).

Thus the NES linear accumulation rule successfully recovers the incubation time strength condition (5) and the Abel type strength condition (7) from their durability diagrams (27) and (29), respectively, while the Robinson accumulation rule does not.

\section{Accumulation rules for Basquin-type durability diagram}

\subsection{General loading}

Consider the case of the durability diagram under constant uniaxial or multiaxial loading $\boldsymbol{\sigma}=$ const starting at $t>0$ described by the power-type relation

$$
|\boldsymbol{\sigma}|=\sigma^{* 0}(\tilde{\boldsymbol{\sigma}} ; t) \text { where } \sigma^{* 0}(\tilde{\boldsymbol{\sigma}} ; t)=\sigma^{0}(\tilde{\boldsymbol{\sigma}}) t^{-1 / b},
$$

Here $b$ is a material constant and $\sigma^{0}=\sigma^{0}(\tilde{\boldsymbol{\sigma}})$ is a material characteristics possibly depending on the unit tensor $\tilde{\boldsymbol{\sigma}}=\boldsymbol{\sigma} /|\boldsymbol{\sigma}|$. Note that (31) is a special case of relation 
(14) with $\sigma^{* *}(t)=t^{-1 / b}$. Durability diagram (31) can be also presented as

$$
t^{* 0}(\boldsymbol{\sigma})=\left(\frac{|\boldsymbol{\sigma}|}{\sigma^{0}(\tilde{\boldsymbol{\sigma}})}\right)^{-b} .
$$

For the uniaxial case, relations (31), (32) can be found e.g. in (Rabotnov, 1966; Penny \& Marriott, 1971; Leckie \& Hayhurst, 1974; Lokoshenko \& Namestnikova, 1983). Note that the Monkman-Grant relation $\dot{\varepsilon}_{m c r} t^{* 0}=$ const also reduces to (32) if the power-type Norton relation is assumed between the applied stress and the minimum creep strain rate $\dot{\varepsilon}_{m c r}$. Similar to fatigue, we will call (31) and (32) the Basquin-type relations.

Then the Robinson rule (1) can be written in form (9), where the corresponding NESF (and NES) has the following form (Mikhailov, 2003),

$$
\underline{\Lambda}^{T R}(\boldsymbol{\sigma} ; t)=\underline{\hat{\Lambda}}^{T R}(\boldsymbol{\sigma} ; t):=\left[\int_{0}^{t} \frac{d \tau}{t^{* 0}(\boldsymbol{\sigma}(\tau))}\right]^{1 / b}=\left[\int_{0}^{t}\left|\frac{\boldsymbol{\sigma}(\tau)}{\sigma^{0}(\tilde{\boldsymbol{\sigma}}(\tau))}\right|^{b} d \tau\right]^{1 / b} .
$$

For a uniaxial process $\sigma(\tau)$, the function $\sigma^{0}(\tilde{\sigma}(\tau))$ is a constant if the stress $\sigma(\tau)$ does not change sign at some instant $\tau$.

On the other hand, the NES $\underline{\hat{\Lambda}}_{\beta}^{T S}\left(\boldsymbol{\sigma} ; t^{\prime}\right)$ given by (21), for durability diagram (31) becomes

$$
\begin{aligned}
\hat{\Lambda}_{\beta}^{T S}\left(\boldsymbol{\sigma} ; t^{\prime}\right) & =\left[\frac{d}{d t^{\prime}} \int_{0}^{t^{\prime}}\left|\frac{\boldsymbol{\sigma}(\tau)}{\sigma^{0}(\tilde{\boldsymbol{\sigma}}(\tau))}\right|^{\beta}\left(t^{\prime}-\tau\right)^{\beta / b} d \tau\right]^{1 / \beta} \\
& =\left[\frac{\beta}{b} \int_{0}^{t^{\prime}}\left|\frac{\boldsymbol{\sigma}(\tau)}{\sigma^{0}(\tilde{\boldsymbol{\sigma}}(\tau))}\right|^{\beta}\left(t^{\prime}-\tau\right)^{\frac{\beta}{b}-1} d \tau\right]^{1 / \beta} \\
& =\left[\int_{-0}^{t^{\prime}}\left(t^{\prime}-\tau\right)^{\beta / b} d\left|\frac{\boldsymbol{\sigma}(\tau)}{\sigma^{0}(\tilde{\boldsymbol{\sigma}}(\tau))}\right|^{\beta}\right]^{1 / \beta} .
\end{aligned}
$$

Particularly, for $\beta=b$ expression (33) implies that

$$
\underline{\hat{\Lambda}}_{b}^{T S}(\boldsymbol{\sigma} ; t)=\underline{\hat{\Lambda}}^{T R}(\boldsymbol{\sigma} ; t) .
$$

This means that for the Basquin durability diagram, the Robinson linear summation rule for partial life-times is a special case of the non-linear rule of NES accumulation (20)-(21) with $\beta=b$.

\section{$5.2 \quad$ Uniaxial step-wise loading}

Consider a uniaxial loading $\sigma(\tau) \geq 0$ such that

$$
\sigma(\tau)= \begin{cases}0, & \tau<t_{0}=0 \\ \sigma_{k}, & t_{k-1} \leq \tau<t_{k}, \quad 0<k<K-1 \\ \sigma_{K}, & t_{K-1} \leq \tau\end{cases}
$$


Under such process, $\sigma^{0}$ is constant in the Basquin durability diagram (32), and the Robinson rule (1) gives the equation

$$
\sum_{k=1}^{K} r_{k}=1
$$

for cumulative durability $t^{*}=t_{K}$, where

$$
r_{k}:=\frac{t_{k}-t_{k-1}}{t^{* 0}\left(\sigma_{k}\right)}=\left(t_{k}-t_{k-1}\right)\left(\frac{\sigma_{k}}{\sigma^{0}}\right)^{b}
$$

are partial life-times. In terms of the $\operatorname{NESF} \underline{\Lambda}^{T R}(\sigma ; t)$, this is equivalent to the strength condition (9), where

$$
\begin{aligned}
\underline{\Lambda}^{T R}(\sigma ; t)= & \underline{\hat{\Lambda}}^{T R}(\sigma ; t)=\underline{\hat{\Lambda}}_{b}^{T S}(\sigma ; t) \\
& =\frac{1}{\sigma^{0}}\left[\sum_{k=1}^{k^{\prime}-1} \sigma_{k}^{b}\left(t_{k}-t_{k-1}\right)+\sigma_{k^{\prime}}^{b}\left(t-t_{k^{\prime}-1}\right)\right]^{1 / b}=\left[\sum_{k=1}^{k^{\prime}-1} r_{k}+r_{k^{\prime}}\right]^{1 / b},
\end{aligned}
$$

$k^{\prime}$ is such that $t_{k^{\prime}-1}<t \leq t_{k^{\prime}}, r_{k^{\prime}}:=\left(t-t_{k^{\prime}-1}\right)\left(\sigma_{k^{\prime}} / \sigma^{0}\right)^{b}$.

On the other hand, for the same process the general power-type NESF by (23) is

$$
\begin{aligned}
& \underline{\Lambda}_{\beta}^{T S}(\sigma ; t)=\max _{0 \leq t^{\prime} \leq t} \underline{\hat{\Lambda}}_{\beta}^{T S}\left(\sigma ; t^{\prime}\right), \\
& \underline{\hat{\Lambda}}_{\beta}^{T S}\left(\sigma ; t^{\prime}\right)=\frac{1}{\sigma^{0}}\left[\sum_{0 \leq t_{k}<t^{\prime}}\left(\sigma_{k+1}^{\beta}-\sigma_{k}^{\beta}\right)\left(t^{\prime}-t_{k}\right)^{\beta / b}\right]^{1 / \beta} .
\end{aligned}
$$

For $\beta=b$, the NESF $\underline{\Lambda}_{b}^{T S}(\sigma ; t)=\underline{\Lambda}^{T R}(\sigma ; t)$ is not sensitive to the load sequence, while $\underline{\Lambda}_{\beta}^{T S}(\sigma ; t)$ is, if $\beta \neq b$. To illustrate this, let us consider the two-step loading, $K=2$, and write (37) for $t=t_{2}>t_{1}$ as

$$
\begin{aligned}
\underline{\Lambda}_{\beta}^{T S}\left(\sigma ; t_{2}\right) & =\max _{t_{1} \leq t^{\prime} \leq t_{2}} \hat{\Lambda}_{\beta}^{T S}\left(\sigma ; t^{\prime}\right), \\
\underline{\hat{\Lambda}}_{\beta}^{T S}\left(\sigma ; t^{\prime}\right) & =\frac{1}{\sigma^{0}}\left[\sigma_{1}^{\beta} t^{\prime \beta / b}+\left(\sigma_{2}^{\beta}-\sigma_{1}^{\beta}\right)\left(t^{\prime}-t_{1}\right)^{\beta / b}\right]^{1 / \beta} \\
& =\left[\left(r_{1}+s^{b} r^{\prime}\right)^{\beta / b}+\left(1-s^{\beta}\right) r^{\prime \beta / b}\right]^{1 / \beta} .
\end{aligned}
$$

where $r^{\prime}=\left(t^{\prime}-t_{1}\right) / t^{* 0}\left(\sigma_{2}\right)$ and $s=\sigma_{1} / \sigma_{2}$ is the parameter of the load sequence, i.e. $s<1$ corresponds to the low-to-high sequence of loading, while $s>1$ to the high-to-low sequence. Particularly, for $\beta=1$ this simplifies to

$$
\begin{aligned}
\underline{\Lambda}_{1}^{T S}\left(\sigma ; t^{\prime}\right) & =\frac{1}{\sigma^{0}}\left[\sigma_{1} t^{1 / b}+\left(\sigma_{2}-\sigma_{1}\right)\left(t^{\prime}-t_{1}\right)^{1 / b}\right] \\
& =\left[\left(r_{1}+s^{b} r^{\prime}\right)^{1 / b}+(1-s) r^{1 / b}\right] .
\end{aligned}
$$


On the other hand, by (36),

$$
\underline{\Lambda}^{T R}\left(\sigma ; t_{2}\right)=\underline{\Lambda}_{b}^{T S}\left(\sigma ; t_{2}\right)=\frac{1}{\sigma^{0}}\left[\sigma_{1}^{b} t_{1}+\sigma_{2}^{b}\left(t_{2}-t_{1}\right)\right]^{1 / b}=\left[r_{1}+r_{2}\right]^{1 / b} .
$$

Equating (38), (39) and (40) to 1, we obtain relations between the partial life times $r_{1}$ and $r_{2}$ in the linear Robinson rule and in the NES nonlinear and linear accumulation rules.

An example is presented in Fig. 4 for $b=5$ and $\beta=1$. All curves are labelled with corresponding value of $s$ and obtained from the linear NES accumulation rule (i.e., $\beta=1$ ), while the straight line $s=1$ is produced also by the Robinson rule. The curves below the straight line give the total life time shorter than predicted by the Robinson rule, while the curves above the straight line give the total life time longer than predicted by the Robinson rule. The high dependence on $s$ shows the high dependence of the durability predictions on the sequence of loading. One can see that the linear NES accumulation rule predicts well the qualitative effects observed experimentally for many materials: shorter life-times than the Robinson rule predicts for low-to-high load sequences, and longer life-times than it predicts for high-to-low load sequences.

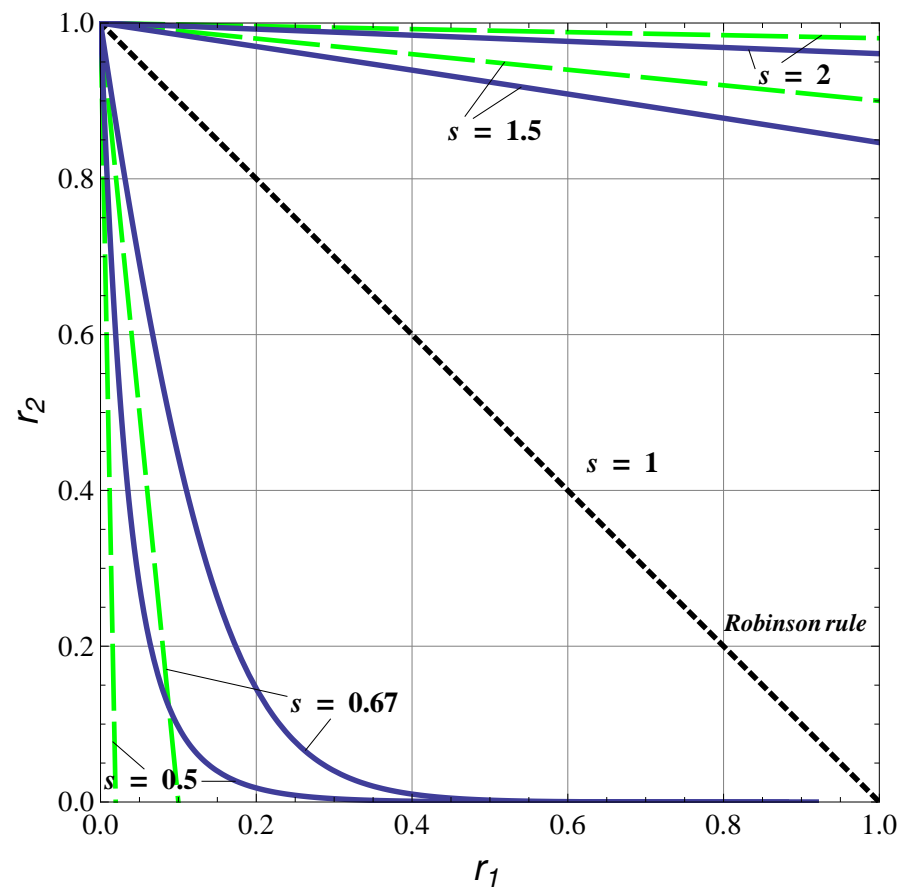

Figure 4: Deviation from the Robinson rule (dotted diagonal straight line) associated with $\underline{\Lambda}_{1}^{T S}(\sigma ; t)$ (curved lines) and with the ad hoc rule (41) (dashed lines), for $b=5$, at several values of $s$.

A simple ad hoc durability criterion is suggested in (Marriott and Penny, 1973), according to which the total durability is estimated as $t_{2}=t^{* 0}\left(\sigma_{2}\right)$, i.e., equal to the durability under the final stress only. By (35) we can reformulate it as

$$
r_{2}=1-s^{-b} r_{1}
$$


which is displayed by the corresponding straight dashed lines in Fig. 4. As we can see from Fig. 4, it deviates not much from the the linear NESF strength condition at small $r_{1}$. However since the criterion $t_{2}=t^{* 0}\left(\sigma_{2}\right)$ effectively replaces the stress on the first step with the stress on the second step, one should not, of course, expect this criterion to be valid for larger $r_{1}$.

\section{Comparison with experiments}

Let us compare some experimental results with the durability predictions given by the Robinson and by the NES accumulation rules.

\subsection{Comparison with two-step experiments by Marriott and Penny, 1973}

In this section we use the durability experiments for an aluminium alloy at $180^{\circ} \mathrm{C}$ under uniaxial constant and variable (step-wise) stress processes reported in (Marriott and Penny, 1973). Fitting the results from (Marriott and Penny, 1973, Table 2) for constant loading to the Basquin durability diagram (32), we obtained the following values for its parameters, $\sigma^{0}=56109 \mathrm{psi} \cdot \mathrm{h}^{1 / b}, b=5.73$.

Fig. 5-10 show the graphs of $\left[\underline{\Lambda}_{1}^{T S}(\sigma ; t)\right]^{b},\left[\underline{\Lambda}_{1}^{T S}(\sigma ; t)\right]^{b}$, given by the linear NES accumulation rule (solid lines) and $\left[\underline{\Lambda}^{T R}(\sigma ; t)\right]^{b}$ given by the Robinson linear accumulation rule (dashed lines) vs. time, calculated from (39) and (40), respectively, for the 2-step low-to-high and high-to-low loading processes from (Marriott and Penny, 1973, Tables 3, 4), where the solid dots show the experimental rupture times. We raised values of $\Lambda$ with the exponent $b$ just to make the graphs for $\left[\underline{\Lambda}^{T R}(\sigma ; t)\right]^{b}$ piecewise linear. The predicted rupture times, to be compared with the experimental data, are given by the intersections of the graphs with the line $\Lambda^{b}=1$. The loading programs are given in the figure captions. The graphs for $\left[\underline{\hat{\Lambda}}_{1}^{T S}(\sigma ; t)\right]^{b}$ and $\left[\underline{\Lambda}_{1}^{T S}(\sigma ; t)\right]^{b}$ coincide except on small parts for $s>1$, i.e. for high-to-low processes, which means that the normalised equivalent stress $\left[\underline{\hat{\Lambda}}_{1}^{T S}(\sigma ; t)\right]^{b}$ can decrease on these parts while the normalised equivalent stress functional $\left[\underline{\Lambda}_{1}^{T S}(\sigma ; t)\right]^{b}$ is always monotonous.

The results from Fig. 5-10 are summarised on Fig. 11 that shows the experimental dots for residual normalized durabilities $r_{2}$ on the second step of the two-step loading processes from the same data by (Marriott and Penny, 1973), the dotted straight line of the durability prediction by the Robinson rule, the curves given by the NES linear rule $(\beta=1)$ and the dashed straight lines given by ad hoc rule (41). Note that the curve given by the NES linear rule for $s=1.43$ overlaps on the graph with the straight line of rule (41) for $s=1.33$. Fig. 12, similarly, shows the same experimental data and the durability prediction by the Robinson rule and by rule (41), but the curves are given by the NES non-linear rule with $\beta=1 / 2$. The auxiliary vertical lines connect the experimental points with the corresponding NES prediction curves (having the same values of $s$ ).

As one can conclude from the data, the Robinson linear rule of partial life-times 


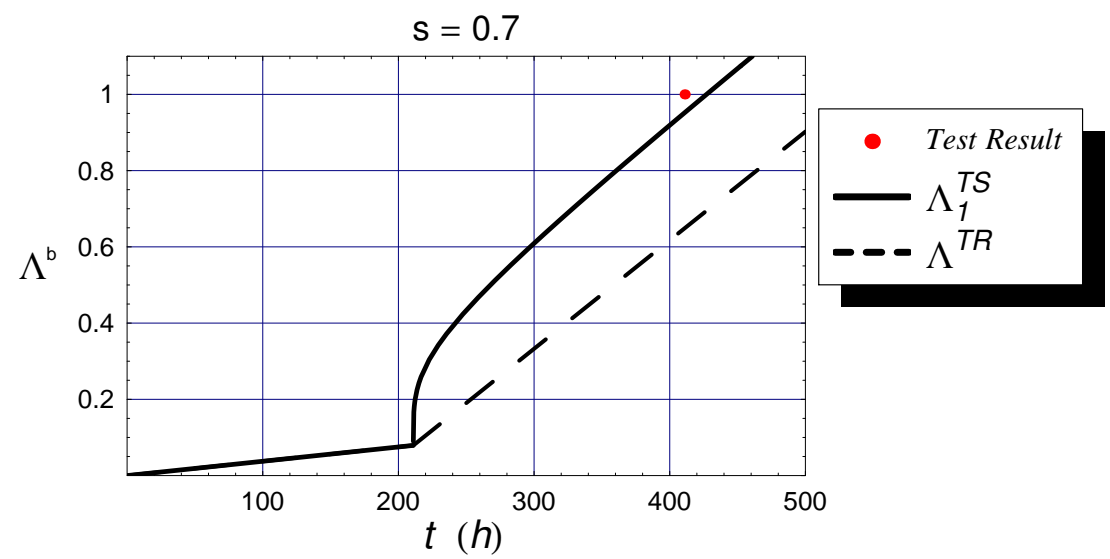

Figure 5: $\left[\underline{\Lambda}_{1}^{T S}(\sigma ; t)\right]^{b}$ and $\left[\underline{\Lambda}^{T R}(\sigma ; t)\right]^{b}$ vs. $t ;[211 \mathrm{~h} @ 14000 \mathrm{psi}+200 \mathrm{~h} @ 20000 \mathrm{psi}]$

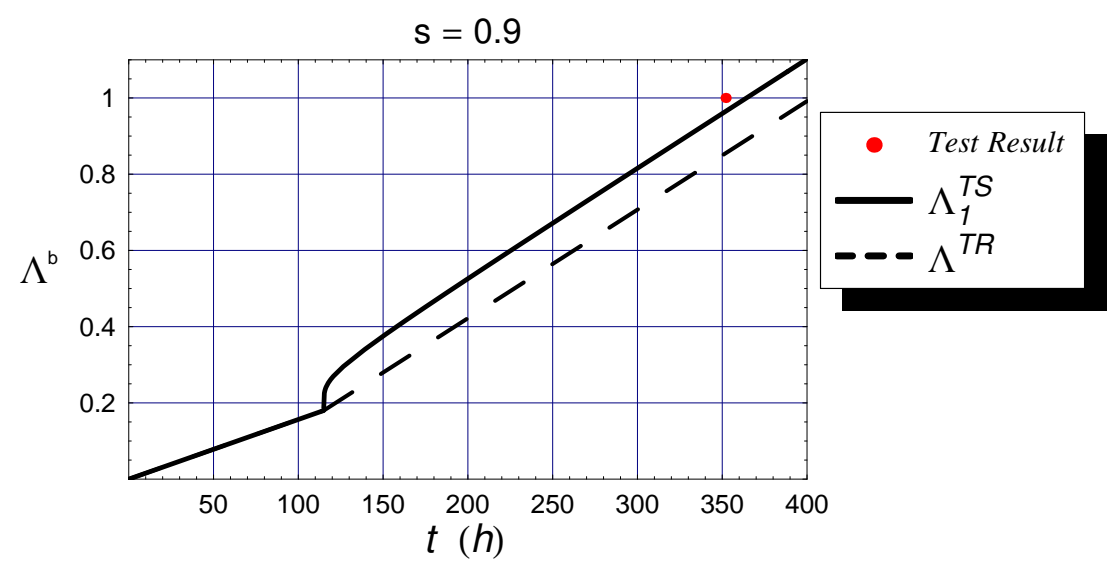

Figure 6: $\left[\underline{\Lambda}_{1}^{T S}(\sigma ; t)\right]^{b}$ and $\left[\underline{\Lambda}^{T R}(\sigma ; t)\right]^{b}$ vs. $t ;[115 \mathrm{~h} @ 18000 \mathrm{psi}+237 \mathrm{~h} @ 20000 \mathrm{psi}]$

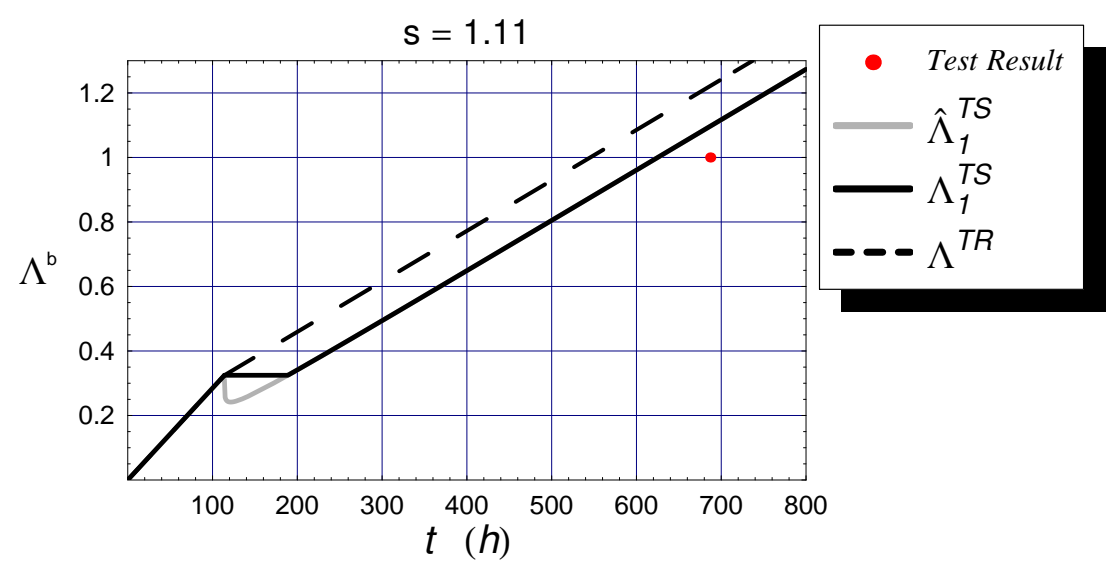

Figure 7: $\left[\underline{\Lambda}_{1}^{T S}(\sigma ; t)\right]^{b}$ and $\left[\underline{\Lambda}^{T R}(\sigma ; t)\right]^{b}$ vs. $t ;[114 \mathrm{~h} @ 20000 \mathrm{psi}+573 \mathrm{~h} @ 18000 \mathrm{psi}]$ accumulation prediction of the residual relative durability $r_{2}$ differs from the experiments up to $73 \%$. Implementation of the linear NES accumulation rule increases 


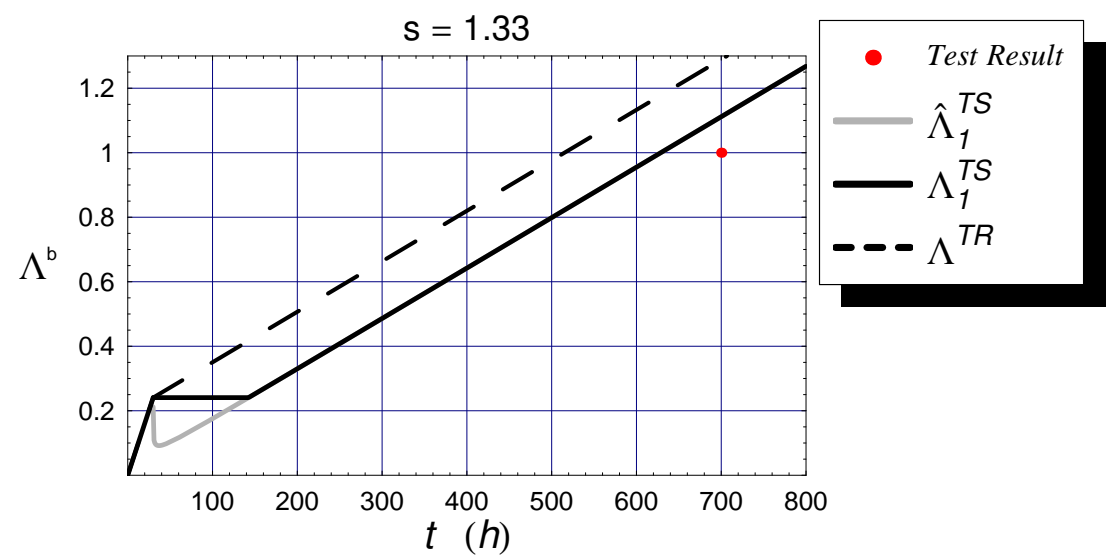

Figure 8: $\left[\underline{\Lambda}_{1}^{T S}(\sigma ; t)\right]^{b}$ and $\left[\underline{\Lambda}^{T R}(\sigma ; t)\right]^{b}$ vs. $t ;[30 \mathrm{~h} @ 24000 \mathrm{psi}+670 \mathrm{~h} @ 18000 \mathrm{psi}]$

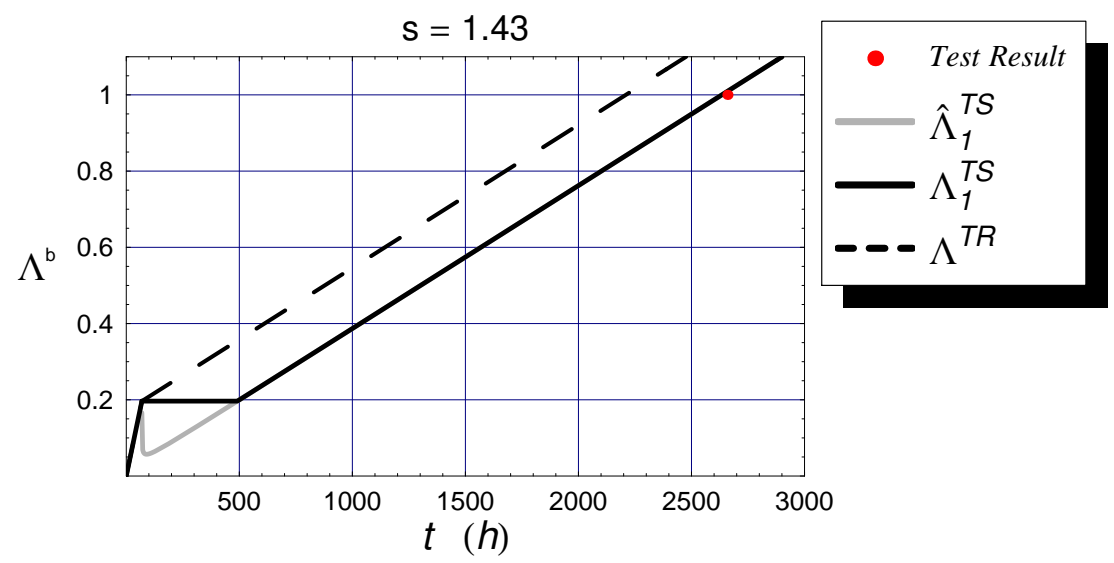

Figure 9: $\left[\underline{\Lambda}_{1}^{T S}(\sigma ; t)\right]^{b}$ and $\left[\underline{\Lambda}^{T R}(\sigma ; t)\right]^{b}$ vs. $t ;[69 \mathrm{~h} @ 20000 \mathrm{psi}+2590 \mathrm{~h} @ 14000 \mathrm{psi}]$

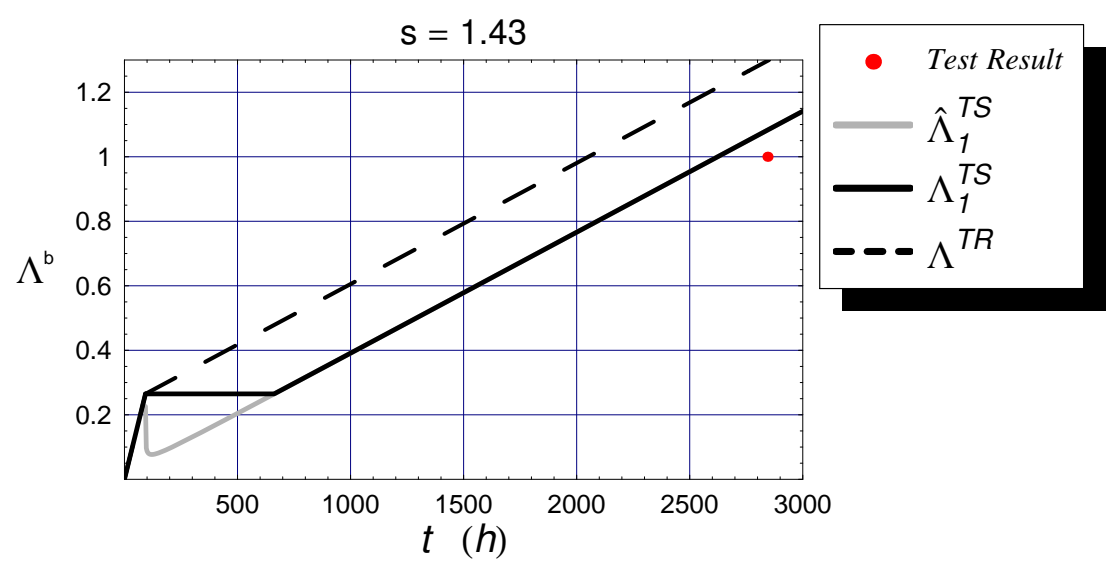

Figure 10: $\left[\underline{\Lambda}_{1}^{T S}(\sigma ; t)\right]^{b}$ and $\left[\underline{\Lambda}^{T R}(\sigma ; t)\right]^{b}$ vs. $t$;

[93h@ $20000 \mathrm{psi}+2751 \mathrm{~h} @ 14000 \mathrm{psi}]$ 


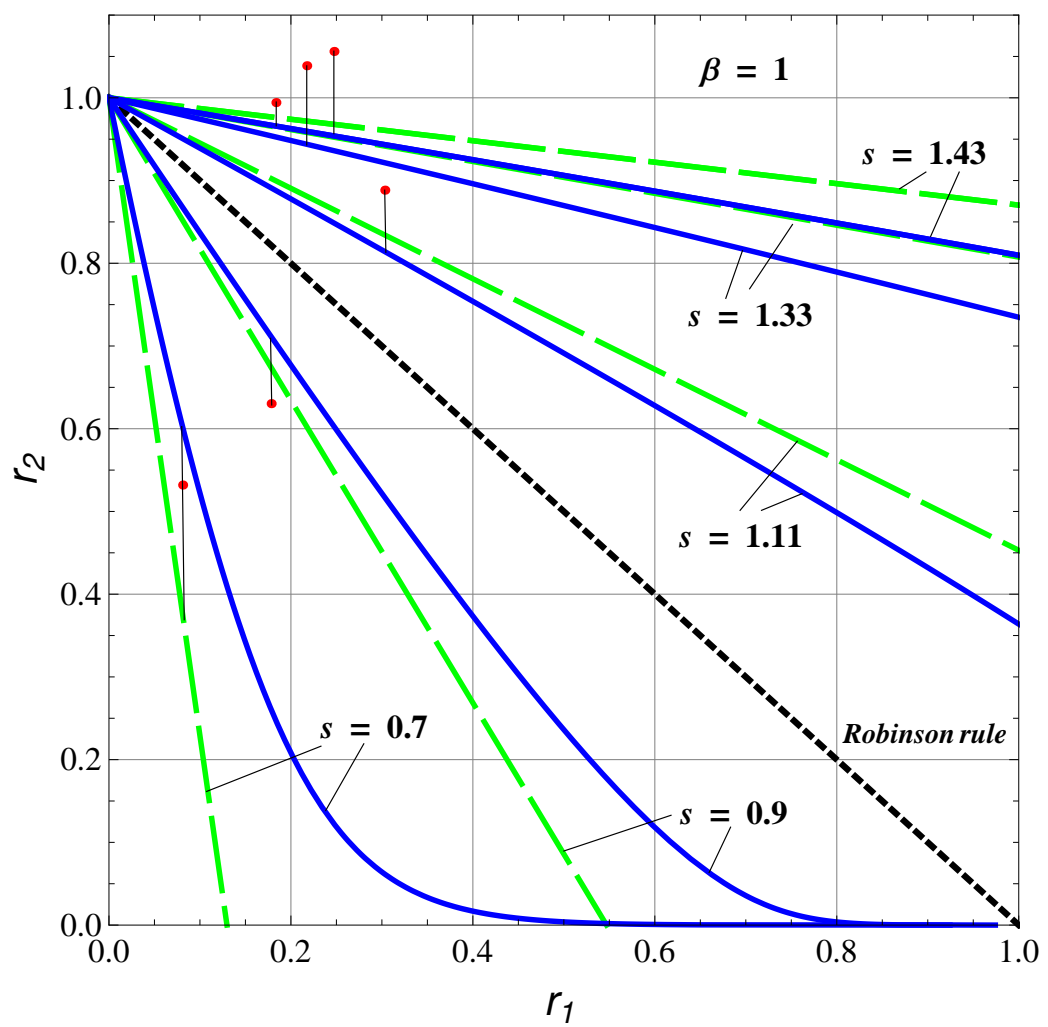

Figure 11: Experimental normalized durabilities $r_{2}$ on step 2 and their predictions by the Robinson rule (dotted diagonal straight line), NES linear rule $(\beta=1)$ (curved lines) and the ad hoc rule (41) (dashed straight lines), for $b=5.73$.

the prediction accuracy from 2 to 6 times making the maximum difference with the experimental durabilities less than $14.3 \%$. The non-linear NES accumulation rule with $\beta=1 / 2$ increases accuracy from 3 to 28 times, making the maximum difference with the experimental durabilities less than $10.3 \%$. Thus the NES accumulation rules with $\beta \leq 1$ provide a viable alternative to the Robinson rule. The ad hoc rule (41) seems to give also a good fit to the experimental data but this can be explained by rather small (up to 0.3) value of the parameter $r_{1}$ for the used experimental data; for higher $r_{1}$ the difference between experiments and the ad hoc rule is expected to increase. 


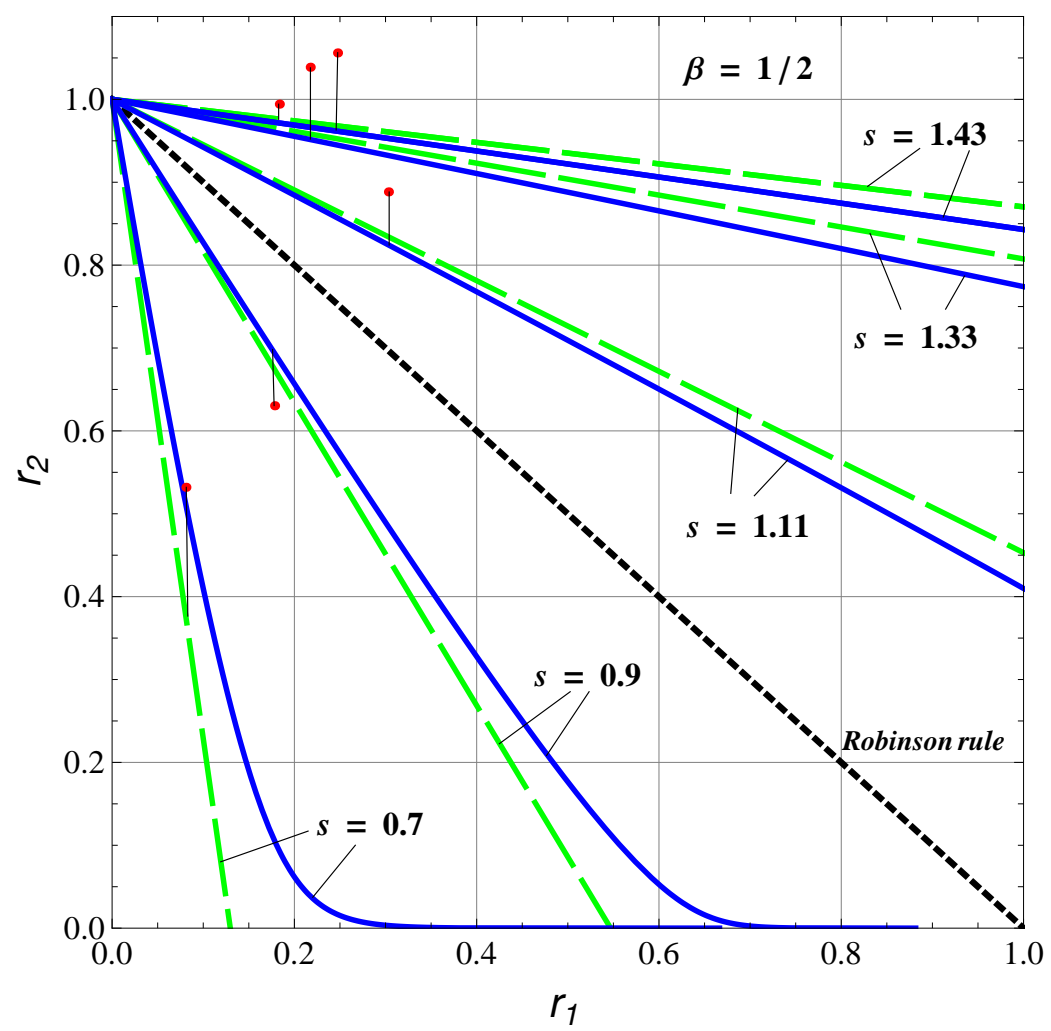

Figure 12: Experimental normalized durabilities $r_{2}$ on step 2 and their predictions by the Robinson rule (dotted diagonal straight line), NES linear rule $(\beta=1 / 2)$ (curved lines) and the ad hoc rule (41) (dashed straight lines), for $b=5.73$.

\subsection{Comparison with multi-step experiments by Goldhoff, 1965}

In this section we use the durability experiments for three types of steel at constant temperatures $1000^{\circ} \mathrm{F}\left(538^{\circ} \mathrm{C}\right)$ or $1050^{\circ} \mathrm{F}\left(566^{\circ} \mathrm{C}\right)$ under uniaxial constant and multistep stress processes reported in (Goldhoff, 1965). Fitting the results from (Goldhoff, 1965, Table 2) for constant loading to the Basquin durability diagram (32), we obtained the following values for its parameters: $\sigma^{0}=63069 \mathrm{psi} \cdot \mathrm{h}^{1 / b}, b=8.17$ for Steel $1 ; \sigma^{0}=71955 \mathrm{psi} \cdot \mathrm{h}^{1 / b}, b=13.4$ for Steel 2 ; and $\sigma^{0}=65412 \mathrm{psi} \cdot \mathrm{h}^{1 / b}, b=11.0$ for Steel 3. These steels were subjected to the following multi-step loading programs, (Goldhoff, 1965, Table 3).

Steel 1, Program 1: 2.7h @ 40000psi + 20.5h @ 35000psi + 52.9h @ 30000psi $+116.2 \mathrm{~h} @ 25000 \mathrm{psi}+213.6 \mathrm{~h} @ 21000 \mathrm{psi}+363.9 \mathrm{~h} @ 30000 \mathrm{psi}$.

Steel 1, Program 2: 719.2h @ 21000psi + 190.2h @ 25000psi + 97.6h@ 30000psi + 4.5h @ 35000psi.

Steel 2:6.7h@50000psi + 42.1h@45000psi + 364.1h@40000psi + 741.5h@ 35000psi+130h@ 45000psi.

Steel 3: 498.3h @ 30000psi + 191.1h @ 32000psi + 5.2h @ 40000psi + 3.6h @ 46000psi+130h@ 45000psi. 
Fig. 13-15 show the graphs of $\left[\hat{\Lambda}_{1}^{T S}(\sigma ; t)\right]^{b},\left[\underline{\Lambda}_{1}^{T S}(\sigma ; t)\right]^{b}$, given by the linear NES accumulation rule (solid lines) and $\left[\underline{\Lambda}^{T R}(\sigma ; t)\right]^{b}$ given by the Robinson linear accumulation rule (dashed lines) vs. time, calculated from (37) for the multi-step loading programs, where the solid dots show the experimental rupture times. As before, we raised values of $\Lambda$ with the exponent $b$ to make the graphs for $\left[\underline{\Lambda}^{T R}(\sigma ; t)\right]^{b}$ piece-wise linear. The predicted rupture times, to be compared with the experimental data, are given by the intersections of the graphs with the line $\Lambda^{b}=1$. Note that similar to Figs. 7-10, the graphs for $\left[\underline{\Lambda}_{1}^{T S}(\sigma ; t)\right]^{b}$ and $\left[\underline{\Lambda}_{1}^{T S}(\sigma ; t)\right]^{b}$ on Fig. 13 (for Program 1) and on Fig. 14 do not coincide, when high-to-low parts of the process are present. We put on the graphs also the dots giving the rupture time predictions by (A) the direct application of the Robinson rule (without approximating the experimental data under constant loading by the Basquin relation (32)), (B) strain hardening theory and (C) strain fraction theory, taken from (Goldhoff, 1965, Table 4).

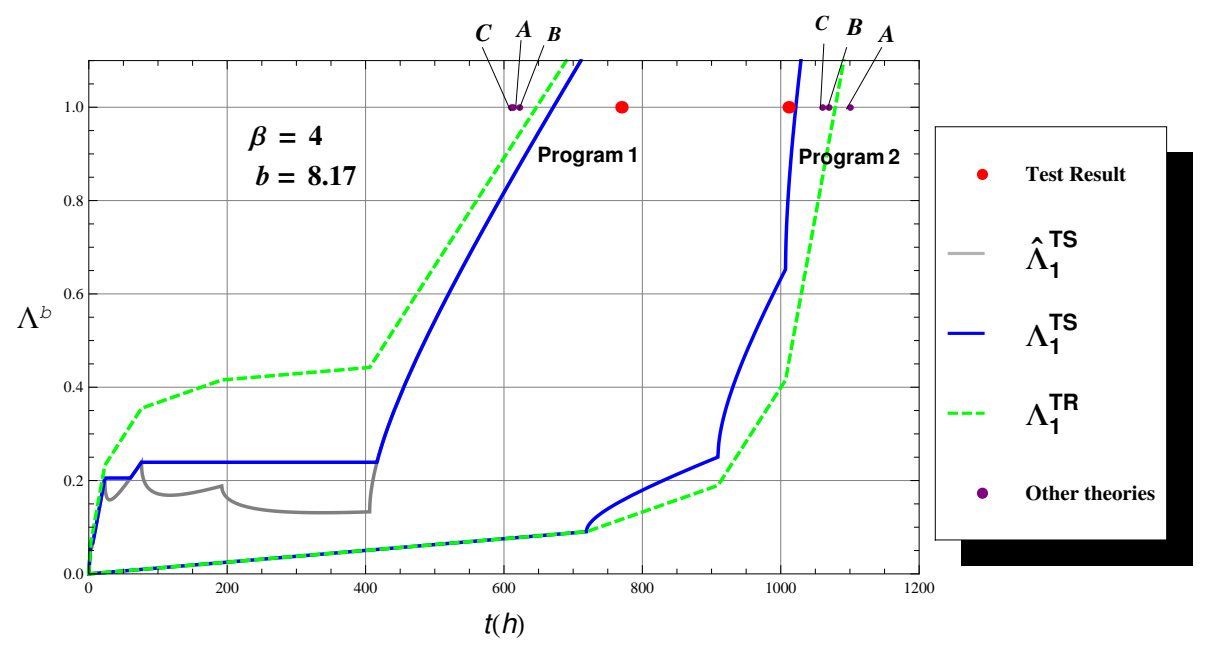

Figure 13: $\left[\underline{\Lambda}_{1}^{T S}(\sigma ; t)\right]^{b}$ and $\left[\underline{\Lambda}^{T R}(\sigma ; t)\right]^{b}$ vs. $t$; comparison with experiment from Goldhoff, 1965, Steel 1, and with other theories.

The linear NES accumulation rule does not fit well to the experimental data, while the nonlinear NES accumulation rules with $\beta=4$ for Steels 1 and 3 and $\beta=2.7$ for Steel 2 give rather good fit, as one can see from the graphs. Implementation of the NES accumulation rule increases the prediction accuracy by $20 \%$ for Program 1 and about 5 times for Program 2 for Steel 1 . Since there is only 1 multi-step test in (Goldhoff, 1965) for each of Steels 2 and 3, the graphs on Figs. 14 and 15 confirm the possibility to find $\beta$ values that fit the experimental rapture times but more data are needed to verify if these values can be taken as material constants. 

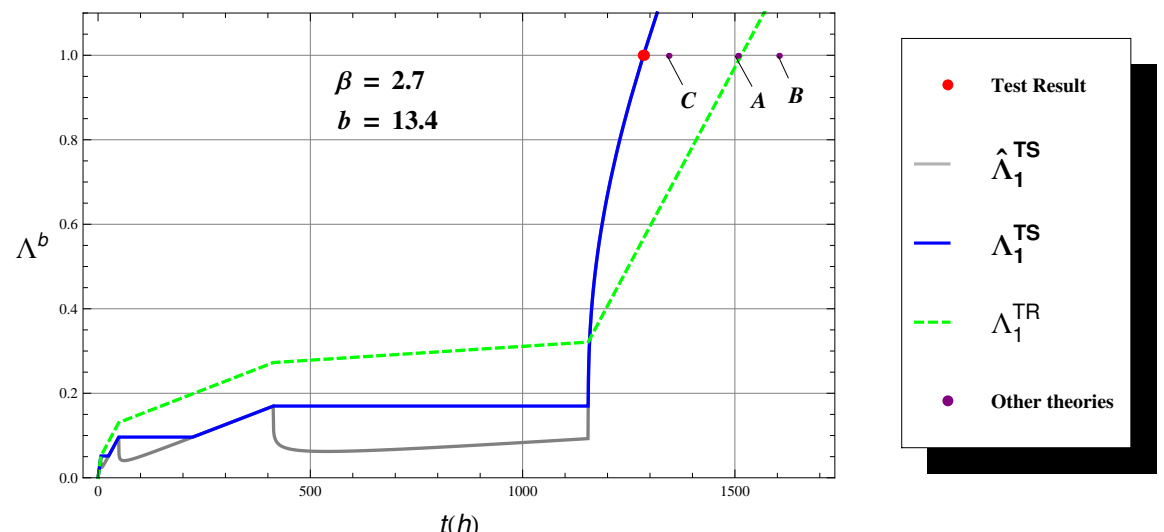

Figure 14: $\left[\underline{\Lambda}_{1}^{T S}(\sigma ; t)\right]^{b}$ and $\left[\underline{\Lambda}^{T R}(\sigma ; t)\right]^{b}$ vs. $t$; comparison with experiment experiment from Goldhoff, 1965, Steel 2, and with other theories.
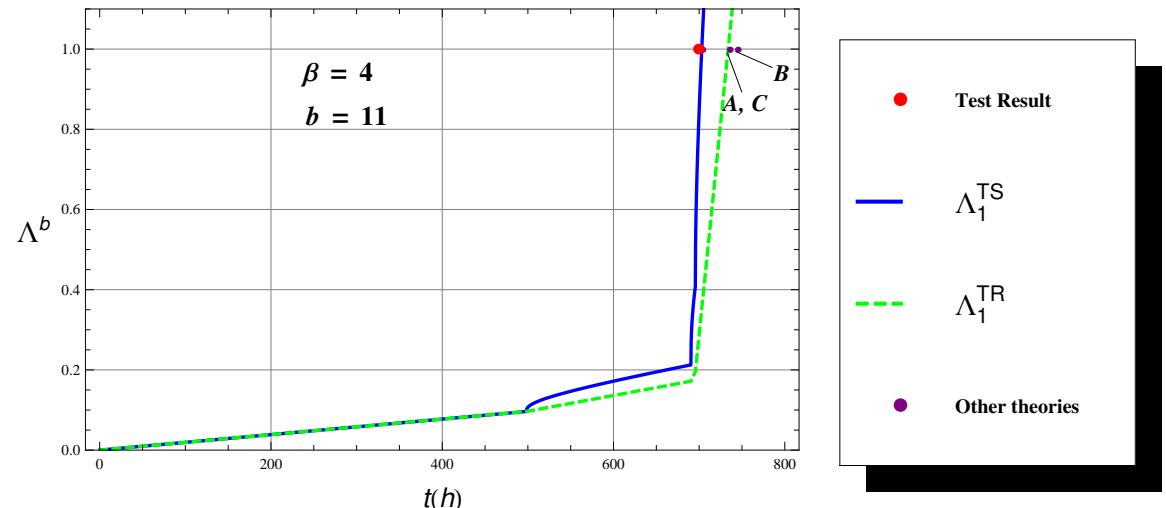

Figure 15: $\left[\underline{\Lambda}_{1}^{T S}(\sigma ; t)\right]^{b}$ and $\left[\underline{\Lambda}^{T R}(\sigma ; t)\right]^{b}$ vs. $t$; comparison with experiment from Goldhoff, 1965, Steel 3, and with other theories.

\section{Conclusion}

The concept of Normalised Equivalent Stress was employed in this paper for the life-time prediction under creep loading. The Robinson linear accumulation rule of partial life times is based on the durability diagram under constant loading. However, the Robinson rule is not sensitive to the loading history, which contradicts some experiments.

We introduced in the paper some accumulation rules for the normalised equivalent stress as an alternative to the Robinson rule. To employ the linear version of the NES accumulation rule for a variable loading, one needs only (a good approximation of) the durability diagram for constant loadings, $\sigma^{* c}(t)$, at least for times $t$ between zero and expected life-time under the variable loading. The nonlinear NES 
accumulation rule used in the paper needs one more material parameter that can be obtained by fitting experimental data.

The linear NES rule allowed to recover the three known temporal strength conditions, considered in the paper, from their corresponding durability diagrams under constant load, while the Robinson rule allowed to do this only for one of them. When compared with some experiments available in the literature, the life time prediction by the NES accumulation rules appeared to be much more accurate than by the Robinson, strain hardening and strain fraction accumulation rules, for these experiments

For the Basquin type durability diagram, the nonlinear NES accumulation rule coincides with the Robinson rule, when the non-linearity parameter $\beta$ equals the Basquin diagram parameter $b$, that shows that the NES approach generalises, in a sense, the Robinson rule complementing it with the loading history sensitivity. This history sensitivity is related with the counting any finite or infinitesimal change of loading in conjunction with the durability diagram starting at the change instant, see e.g. (18), (19), (23), which might be the key in explanation of the higher accuracy of the NES approach.

In this paper the NES accumulation rules were implemented in total temporal strength conditions (rupture criteria). But they can be equally employed in local or nonlocal strength conditions generalising in the latter the approaches analysed in (Mikhailov 1995a, 1995b; Isupov \& Mikhailov 1998)) at a stress concentrator, particularly for crack propagation analysis, cf. (Mikhailov and Namestnikova, 2003, 2004).

The developed NES accumulation rules can be used not only for creep but also for dynamic strength predictions and can be modified to describe the fatigue durability, cf. (Mikhailov and Namestnikova, 2003, 2004).

\section{APPENDIX}

\section{Normalised Equivalent Stress Definition and Properties}

For a given process $\boldsymbol{\sigma}(\tau)$ and an instant $t$, the temporal normalised equivalent stress functional is defined in (Mikhailov, 2003) as infimum of numbers $\Lambda^{\prime}>0$ such that there is no rupture at or before the time $t$ under the process $\frac{1}{\Lambda^{\prime \prime}} \boldsymbol{\sigma}(\tau)$ for any $\Lambda^{\prime \prime}>\Lambda^{\prime}$; if there is no such $\Lambda^{\prime}$, we assign $\underline{\Lambda}^{T}(\boldsymbol{\sigma} ; t)=\infty$.

So defined, the temporal normalised equivalent stress functional $\underline{\Lambda}^{T}$ is a material characteristics and should be identified from experiments and/or a model. The definition implies that the functional $\underline{\Lambda}^{T}(\boldsymbol{\sigma} ; t)$ is non-negative positively-homogeneous of the order +1 in the first argument and non-decreasing in the second argument, that is

$$
\underline{\Lambda}^{T}(k \boldsymbol{\sigma} ; t)=k \underline{\Lambda}(\boldsymbol{\sigma} ; t) \geq 0 \text { for any } k>0, \quad \underline{\Lambda}^{T}\left(\boldsymbol{\sigma} ; t^{\prime \prime}\right) \geq \underline{\Lambda}^{T}\left(\boldsymbol{\sigma} ; t^{\prime}\right) \text { if } t^{\prime \prime}>t^{\prime} .
$$

These properties make $\underline{\Lambda}^{T}$ uniquely determinable and narrow down the admissible forms of $\underline{\Lambda}^{T}$, to be approximated from experimental data. 


\section{References}

Bui-Quoc, T., 1979, An engineering approach to cumulative damage in metals under creep loading. Journal of Engineering Materials and Technology 101, 337343.

Goldhoff, R.M., 1965, Uniaxial creep-rapture behaviour of low-alloy steel under variable loading conditions. Trans. ASME, J. Basic Engineering 87, 374-378.

Gomuc, R., Bui-Quoc, T., 1986, An analysis of the fatigue/creep behavior of 304 stainless steel using a continuous damage approach. ASME Journal of Pressure Vessel Technology 108, 280-288.

Il'ushin, A., 1967, About one theory of durability. Mech. of Solids (Izv. AN SSSR. MTT) 2(3), 21-35.

Isupov, L.P., Mikhailov, S.E., 1998, Comparative analysis of several non-local fracture criteria. Archive of Applied Mechanics 68, 597-612.

Kashtanov, A.V., Petrov, Y.V., 2007, Kinetic description of incubation processes under dynamic fracture. Doklady Physics 52(5), 270-273.

Kashtanov, A.V., Petrov, Y.V., Pugno, N., Carpinteri, A., 2008, Dynamic fracture as a process of nonlinear damage wave propagation. Int. J. Fracture 150, 227-240.

Leckie, F.A., Hayhurst, D.R., 1974, Creep rupture of structures. Proceedings of the Royal Society of London. Series A, Mathematical and Physical Sciences 340(1622), 323-347.

Lokoshchenko, A., Namestnikova, I., 1983, Description of long-term strength under load applied in steps. Strength of Materials 15(1), 8-13.

Marriott, D., Penny, R., 1973, Strain accumulation and rupture during creep under variable uniaxial tensile loading. Journal of Strain Analysis 8(3), 151-159.

Mikhailov, S.E., 1995a, A functional approach to non-local strength conditions and fracture criteria - I. Body and point fracture. Engineering Fracture Mechanics 52, $731-743$.

Mikhailov, S.E., 1995b, A functional approach to non-local strength conditions and fracture criteria - II. Discrete fracture. Engineering Fracture Mechanics 52, $745-754$.

Mikhailov, S.E., 2003, Theoretical backgrounds of durability analysis by normalized equivalent stress functionals. Mathematics and Mechanics of Solids 8, 105-142.

Mikhailov, S.E., Namestnikova, I.V., 2003, Fatigue strength and durability analysis by normalised equivalent stress functionals. In: Proceedings of the 9th International Conference on the Mechanical Behaviour of Materials, ICM9, 2003, Geneva, pp. $1-10$. 
Mikhailov, S.E., Namestnikova, I.V., 2004, Local and non-local normalised equivalent strain functionals for cyclic fatigue. In: Proceedings of the Seventh International Conference on Biaxial/Multiaxial Fatigue \& Fracture, DVM, Berlin, pp. 409-414.

Mikhailov, S.E., Namestnikova, I.V., 2009, About accumulation rules for life time prediction under variable loading. In: Proceedings of the 12th International Conference on Fracture (CD), Natural Resources Canada \& National Research Council of Canada, Ottawa, Canada, 12-17 July, pp. 1-10.

Morozov, N., Petrov, Y., 2000, Dynamics of Fracture. Springer, Berlin-Heidelberg.

Nikiforovsky, V., 1976, On kinetic character of brittle fracture of solids. Appl. Mech. and Techn. Phys. 18(5), 150-157.

Nikiforovsky, V., Shemyakin, E., 1979, Dynamic Fracture of Solids. Novosibirsk Univ. Press, Novosibirsk.

Penny, R., Marriott, D., 1971, Design for Creep. McGraw-Hill, London.

Rabotnov, Y.N., 1969, Creep Problems in Structural Members. North-Holland Publ., Amsterdam-London, Russian edition: Nauka, Moscow, 1966.

Robinson, E., 1938, Effect of temperature variation on creep strength of steels. Trans. ASME 60, 253-259.

Robinson, E., 1952, The effect of temperature variation on the long-time rupture strength of steels. Trans. ASME 74, 777-780.

Suvorova, Y.V., 1979, About strength criterion based on damage accumulation and its application to composites. Mechanics of Solids (Izv. AN SSSR, MTT) 14(4), 9295, (original Russian edition: p. 107-111).

Suvorova, Y.V., Viktorova, I.V., Mashinskaya, G.P., 1980, Long-time failure of inelastic composites. Mechanics of Composite Materials 15(5), 516-519, (original Russian edition: 1979, No. 5, 794-798). 